\title{
Intergenerational implications of alcohol intake: metabolic disorders in alcohol-naïve rat offspring
}

\author{
Pawel Mierzejewski $^{1}{ }^{1}$, Alicja Zakrzewska ${ }^{1}$, Julita Kuczyńska ${ }^{1}$, Edyta Wyszogrodzka ${ }^{1}$, Monika Dominiak ${ }^{\text {Corresp. } 1}$ \\ ${ }^{1}$ Department of Pharmacology, Institute of Psychiatry and Neurology, Warsaw, Poland \\ Corresponding Author: Monika Dominiak \\ Email address: mdominia@wp.pl
}

Alcohol drinking may be associated with an increased risk of various metabolic diseases. Rat lines selectively bred for alcohol preference and alcohol avoidance constitute an interesting model to study inherited factors related to alcohol drinking and metabolic disorders. The aim of the present study was to compare the levels of selected laboratory biomarkers of metabolic disorders in blood samples from naïve offspring of Warsaw alcohol high-preferring (WHP), Warsaw alcohol low-preferring (WLP), and wild Wistar rats. Blood samples were collected from 3-month old (300-350 g) alcohol-naïve, male offspring of WHP $(n=8)$ and WLP rats $(n=8)$, as well as alcohol-naïve, male, wild Wistar rats. Markers of metabolic, hepatic, and pancreatic disorders were analysed (levels of homocysteine, glucose, total cholesterol, triglycerides and y-glutamyl transferase (GGT), aspartate (AST), alanine aminotransferase (ALT), and amylase serum activities). Alcohol-naïve offspring of WHP, WLP, and wild Wistar rats differed significantly in the levels of triglycerides, total cholesterol, homocysteine, as well as in the activity of GGT, ALT, AST, and amylase enzymes. Most markers in the alcohol-naïve offspring of WHP rats were altered even thought they were never exposed to alcohol pre- or postnatally. This may suggest that parental alcohol abuse can have a detrimental influence on offspring vulnerability to metabolic disorders. 
1 Intergenerational implications of alcohol intake: metabolic disorders in

2 alcohol-naïve rat offspring

3

4

5 Paweł Mierzejewski ${ }^{1}$, Alicja Zakrzewska ${ }^{1}$, Julita Kuczyńska ${ }^{1}$, Edyta Wyszogrodzka ${ }^{1}$, Monika

6 Dominiak $^{1^{*}}$

7

$8 \quad{ }^{1}$ Department of Pharmacology, Institute of Psychiatry and Neurology, Warsaw, Poland

9

10

11

12

13

14

15

16 "Corresponding author:

17 Monika Dominiak

18 ul. Sobieskiego 9, 02-957 Warsaw, Poland

19 email: $\underline{\text { mdominia@wp.pl }}$

20

21 


\section{Abstract}

24 Alcohol drinking may be associated with an increased risk of various metabolic diseases. Rat

25 lines selectively bred for alcohol preference and alcohol avoidance constitute an interesting 26 model to study inherited factors related to alcohol drinking and metabolic disorders. The aim of

27 the present study was to compare the levels of selected laboratory biomarkers of metabolic 28 disorders in blood samples from naïve offspring of Warsaw alcohol high-preferring (WHP),

29 Warsaw alcohol low-preferring (WLP), and wild Wistar rats. Blood samples were collected from 30 3-month old (300-350 g) alcohol-naïve, male offspring of WHP $(\mathrm{n}=8)$ and WLP rats $(\mathrm{n}=8)$, as

31 well as alcohol-naïve, male, wild Wistar rats. Markers of metabolic, hepatic, and pancreatic 32 disorders were analysed (levels of homocysteine, glucose, total cholesterol, triglycerides and $\gamma$ 33 glutamyl transferase (GGT), aspartate (AST), alanine aminotransferase (ALT), and amylase 34 serum activities). Alcohol-naïve offspring of WHP, WLP, and wild Wistar rats differed 35 significantly in the levels of triglycerides, total cholesterol, homocysteine, as well as in the 36 activity of GGT, ALT, AST, and amylase enzymes. Most markers in the alcohol-naïve offspring 37 of WHP rats were altered even thought they were never exposed to alcohol pre- or postnatally.

38 This may suggest that parental alcohol abuse can have a detrimental influence on offspring 39 vulnerability to metabolic disorders. 


\section{Introduction}

Heavy alcohol drinking can lead to serious health problems, including cardiovascular diseases (CVD) and liver cirrhosis [1-3]. In numerous studies, heavy alcohol drinking was linked to both an increased risk of CVD and metabolic syndrome, leading to the hypothesis that alcohol-induced metabolic disorders could stand behind the relationship between excessive

51 alcohol consumption and CVD [4-6]. The above-mentioned associations may be related to environmental as well as genetic factors. Twin, family, and adoption studies have consistently shown that genetic factors play an important role in the development of alcohol dependence [79]. Genetic loading is also an important risk factor for dyslipidaemia, diabetes, and metabolic syndrome [10-12]. Hence, one may hypothesise that excessive alcohol consumption and metabolic abnormalities are related to the same genetic mechanism(s).

Prenatal alcohol consumption has been shown to interfere with the development of multiple organs and systems, resulting in Foetal Alcohol Spectrum Disorders (FASD) [13]. Epidemiological studies have revealed that alcohol is the most common teratogen to which humans are exposed. It was estimated that 8 children per 1,000 live births are diagnosed with some degree of FASD [14]. This condition is characterised by a spectrum of structural defects, central nervous system disorders, as well as growth deficits, and metabolic disorders $[15,16]$. Although it is perceived as a childhood disorder, the majority of health problems, including growth restriction, hyperinsulinemia, and other metabolic disruptions are lifelong, persisting into adulthood $[17,18]$.

Apart from this well-known unfavourable effect on the foetus, recent studies on rats have

67 highlighted the possible negative impact on offspring of maternal alcohol exposure also around the time of conception. Several lines of evidence have suggested that periconceptional alcohol 
69 use may have a negative impact on adult offspring, including increased obesity risk, altered

70 plasma lipids, leptin profiles, and liver steatosis [19]. Furthermore, the study of Dorey et al.

71 (2018) [20] revealed a greater risk of obesity in the offspring of females exposed to alcohol

72 around the time of conception. It was associated with the altered expression of key components

73 of the mesolimbic reward pathway and an increased preference for palatable foods in offspring.

74 However, apart from reports on maternal alcohol use during pregnancy or in the

75 periconceptional period, studies on paternal preconception alcohol exposure on offspring also

76 provide interesting findings. Paternal alcohol intake during the preconception period induces a

77 spectrum of health problems and alters gene expression in rodent offspring [21-24]. The results

78 of the study of Kim et al. (2014) [25] suggest that the preconceptional exposure to ethanol of

79 male mice before mating induces ADHD-like behaviours in offspring, possibly via epigenetic

80 changes in gene expression. Asimes et al. (2017) [26] demonstrated that adolescent binge ethanol

81 exposure altered DNA methylation patterns in the hypothalamus of alcohol-naïve offspring. In

82 another study examining parental binge alcohol abuse, differences in the expression of genes

83 involved in neurogenesis, reproductive function, and regulation of obesity were observed in

84 offspring [27]. Also, the studies of Chang et al. (2019a, 2019b) [28,29] using a mouse model of

85 paternal alcohol exposure have identified the markers of hepatic fibrosis and abnormalities in

86 both lipid production and insulin signaling in the offspring of alcohol-exposed sires. The

87 aforementioned studies raise the possibility that alcohol affects naïve offspring even in the

88 absence of direct foetal alcohol exposure, and epigenetic inheritance may be the mechanism

89 responsible for this phenomenon.

90 In light of the metabolic changes associated with alcohol consumption, whether common

91 metabolic markers differ in alcohol-naïve offspring of high- and low-preferring rats is of interest. 
92 Rat lines selectively bred for alcohol preference and alcohol avoidance constitute an interesting

93 model to study the inherited factors related to alcohol drinking and metabolic disorders. The

94 breeding of rats was carried out as previously described by Rok-Bujko et al. (2006) [30].

95 Specifically, Warsaw high-preferring (WHP) and Warsaw low-preferring (WLP) rats were bred

96 from Wistar rats for opposite ethanol preference [30,31]. When given a choice between water

97 and $10 \%$ ethanol, WHP rats voluntarily drink excessive amounts of ethanol $(>5.0 \mathrm{~g} / \mathrm{kg} / 24 \mathrm{~h})$,

98 while WLP rats drink marginal quantities of ethanol [31].

99 The aim of the present study was to compare the common markers reflecting 100 cardiovascular, liver, and pancreatic function in alcohol-naïve offspring of WHP, WLP, and

101 Wistar rats: the levels of homocysteine, glucose, total cholesterol, triglycerides, $\gamma$-glutamyl 102 transferase (GGT), aspartate aminotransferase (AST), alanine aminotransferase (ALT), and 103 amylase serum activities. We hypothesise that markers of cardiovascular, liver, and pancreatic 104 function differ between alcohol-naïve offspring of WHP, WLP, and Wistar rats.

109 Materials and methods

\section{Rats and experimental design}

111 Twenty-four (8 per group), twelve-week-old, alcohol-naïve Warsaw high-preferring

112 (WHP), alcohol-naïve Warsaw low-preferring (WLP) rats, and male albino Wistar rats (Charles

113 River, Sulzfeld, Germany) were used for the study. The WLP and WHP rats were obtained from 
114 the Department of Pharmacology and Physiology of the Nervous System at the Institute of

115 Psychiatry and Neurology in Warsaw, Poland.

116 During the selection, which is a standardized procedure repeated in every generation,

117 WHP and WLP rats were exposed to alcohol for 30 days, after reaching a weight of $250 \mathrm{~g}$. In

118 males, this occurs at around 60 days of age. When given a choice between water and $10 \%$

119 ethanol, WHP rats voluntarily drank excessive amounts of ethanol $(>5.0 \mathrm{~g} / \mathrm{kg} / 24 \mathrm{~h})$, while WLP

120 rats consumed negligible quantities of ethanol [31]. Periods of binge drinking are observed only

121 in WHP rats. In this study, all parents of examined offspring were previously subject to a

122 standardized selection procedure. After a 30-day period of alcohol exposure, they were mated 24

123 hours after the last ethanol consumption and subsequent offspring were produced. The parents

124 were not intoxicated at the time of mating and were not exposed to alcohol at any time during

125 pregnancy, thus, the analysed offspring were never directly exposed to alcohol. Two rats per

126 litter were used (4 litters in total for each group).

127 All the rats were housed in the same environmental conditions and handled by the same 128 experimenters for at least 30 days before blood sampling. The environmental conditions were as 129 previously described in the study of Zakrzewska et al. (2019) [32]. Specifically, the rats were

130 housed in standard plastic cages, in groups of three, with wood chip bedding on the floor, in fully 131 controlled environmental conditions: temperature $22 \pm 2{ }^{\circ} \mathrm{C}$, humidity $50 \% \pm 5$, and 12 -hour 132 light/12-hour dark cycles with lights on at 8.00 a.m. All the rats had unlimited access to tap water 133 and standard laboratory chow (Labofeed H, WPIK, Kcynia, Poland) throughout the duration of 134 the study.

135 The offspring of all rat lines were weighed at 21, 60, and 90 days of age. There were no 136 differences in body weight between the tested rat lines at any stage of their development or their 
137 food intake (both $\mathrm{F}<1.0, \mathrm{p}>0.05$ ). In the last measurement at 90 days of age (at the time of

138 sampling) the offspring of WHP, WLP, and Wistar rats weighed $345 \mathrm{~g}( \pm 27.6), 338 \mathrm{~g}( \pm 30.5)$

139 and $341 \mathrm{~g}( \pm 25.8)$, respectively.

140 Blood samples were obtained from 3-month-old (average of 300-350 g) drug- and

141 alcohol-naïve male offspring of WHP $(n=8)$ and WLP rats $(n=8)$. Non-selected 3-month-old,

142 drug- and alcohol-naïve male Wistar rats (Charles River, Sulzfeld, Germany; 300-350 g; n=8)

143 were used as additional controls. The rats were deprived of food for $12 \mathrm{~h}$ overnight and were

144 sacrificed by rapid decapitation with a guillotine designed for rodents. Trunk blood was collected

145 into a tube immediately after decapitation. Serum was obtained after centrifugation at $1600 \mathrm{~g}$ for

14615 minutes. The serum was collected into a plain tube and stored at $-80^{\circ} \mathrm{C}$ until the analysis. No

147 haemolysis was observed in any collected sample.

148 The Ethics Committee for Animal Care provided its consent for the study (Agreement no.

149 10/2010). The study was performed in full compliance with respective Polish and European

150 ethical regulations (Directive No. 86/609/EEC).

151

\section{Biochemical analysis}

153

The activities of AST, ALT, GGT, and amylase, as well as the levels of glucose, total

154 cholesterol, and triglycerides were measured in the serum using automated enzymatic

155 colorimetric methods (Cobas Integra, Roche Diagnostics, Rotkreuz, Switzerland). Serum

156 homocysteine determination was carried out using fully automated chemiluminescence assay

157 (ADVIA Centaur, Siemens Healthcare Diagnostics, Erlangen, Germany). The assays were

158 performed in accordance with the manufacturers' instructions in the analytical laboratory of the

159 Institute of Psychiatry and Neurology in Warsaw. 


\section{Statistical analysis}

A one-way ANOVA was used to evaluate between-group differences in the biochemical

163 measures. The Newman-Keuls test was applied for post hoc comparisons. The normality of

164 distribution of the tested variables was verified using the Shapiro-Wilk test. For two tested 165 variables: ALT and triglycerides, the distribution could not be considered as normal. For the 166 analysis of these parameters, the Kruskal-Wallis test was applied, while Dunn's multiple 167 comparisons test was used for post hoc comparisons. P-values of less than 0.05 were considered 168 significant. The study was exploratory in nature, thus, no correction for multiple comparisons 169 was applied. The Statistica 12.0 software package (StatSoft, Inc., Tulsa, OK, USA) was used to 170 analyse all the data.

\section{Results}

The one-way ANOVA revealed that the offspring of WHP, WLP, and Wistar rats

177 differed significantly in the following laboratory parameters: homocysteine $(F(2,21)=9.65$, $178 \mathrm{p}=0.001)$, total cholesterol $(\mathrm{F}(2,21)=44.63, \mathrm{p}<0.001)$, AST $(\mathrm{F}(2,21)=6.74, \mathrm{p}=0.005)$, amylase $(\mathrm{F}(2,21)=14.30, \mathrm{p}<0.001)$, and $\operatorname{GGT}(\mathrm{F}(2,21)=135.87, \mathrm{p}<0.001)$ (Table 1). There were no

180 differences in blood glucose levels $(\mathrm{F}(2,21)=0.86, \mathrm{p}=0.437)$.

181

182 [Table 1] 
The post hoc Newman-Keuls comparisons indicated that the alcohol-naïve offspring of

184 WHP rats had significantly higher levels of total cholesterol and homocysteine, as well as

185 significantly higher AST and amylase activity as compared to the alcohol-naïve WLP and the

186 Wistar controls. The offspring of WHP rats had a significantly higher level of homocysteine as

187 compared to the offspring of WLP rats, but not the Wistar rats. Surprisingly, the offspring of

188 WHP subjects showed significantly lower GGT activity as compared to the WLP and the Wistar 189 rats.

190 Regarding ALT as well as triglycerides, the Kruskal-Wallis test revealed a highly

191 significant difference between the tested groups: $(H(2)=17.6, p<0.001,(H(2)=20.2, p<0.001$,

192 respectively. Post hoc comparisons for these markers revealed significant differences between

193 WHP and Wistar rats (both $\mathrm{p}<0.001$ ) for both markers, as well as between WHP and WLP rats

$194(\mathrm{p}=0.04, \mathrm{p}=<0.001$, for ALT and triglycerides, respectively).

\section{Discussion}

This is the first study to show changes in selected biochemical markers of metabolic

199 disorders in naïve Wistar rats selected for high alcohol preference (WHP rats). A major finding

200 of the present study is that the alcohol-naïve offspring of WHP rats have significantly higher

201 blood levels of total cholesterol, triglycerides, and homocysteine than their WLP counterparts

202 and wild Wistar rats. Some differences were also observed in the markers of hepatic and 203 pancreatic function between alcohol-naïve offspring of WHP, WLP, and Wistar rats (ALT, AST, 204 and amylase were increased, whereas GGT activity was decreased in WHPs). 
206 obesity and hypertension - in alcohol-naïve WHP rats as compared to alcohol-naïve WLP rats

207 [33]. Although leptin is involved in lipid metabolism [34], the exact mechanism of the above 208 observation in alcohol-naïve WHP rats remains unclear. In other studies, chronic alcohol 209 exposure in rodents was shown to reduce the serum level of leptin [35]. Furthermore, leptin 210 deficiency was associated with the development of fatty liver in mice [36].

211 A high level of total cholesterol or triglycerides is associated with an increased risk of 212 atherosclerosis, myocardial infarction, and stroke [37-39]. Elevated levels of homocysteine are 213 thought to facilitate the development of atherosclerosis and are considered a risk factor for 214 coronary disease and stroke [40-41]. Several studies have shown a positive correlation between 215 the amount of alcohol consumption and the blood homocysteine level [42-43]. It has also been 216 shown that active drinking and early abstinent alcoholics have a higher level of homocysteine in 217 the serum as compared to the general population [44-45]. There are also some epidemiological 218 analyses showing a link between familial alcoholism and obesity among men and women [46]. There are many hypotheses aimed at explaining the increased risk of metabolic diseases in alcoholics. Our study indicates that some inherited factors (genetic, epigenetic) may play a role. These factors transmitted from alcoholic parents to their offspring could, at least partially, be responsible for the increased risk of metabolic disorders. A few studies have attempted to 223 identify the genes responsible for the differences in alcohol preference among the naïve offspring

224 of high- and low-preferring rats [47-49]. One such study indicated that alcohol preference may 225 share some genetic factors with the cardiovascular system and metabolism [49], however, this 226 study failed to identify any gene differences between high- and low-preferring rats. Thus, it does 227 not support the hypothesis that the observed phenotypic differences are gene related. One can 
228 hypothesise that other factors, for example, the epigenetic mechanism, may have a dominant role

229 in the observed phenomenon. Many reports in the literature have indicated that environmental

230 factors can have long-term effects on gene expression through epigenetic mechanisms [50].

231 Epigenetics refers to mechanisms that modify gene expression without any changes in the DNA

232 sequence. Recent evidence suggests that epigenetic modifications can be carried to the offspring

233 via the germline and are responsible for the transmission of alcohol-related disorders across

234 generations [51-52]. Interestingly, there are studies showing the association of parental alcohol

235 intake with subsequent changes in gene expression in naïve offspring [24-26], as well as with

236 changes in lipid metabolism [27]. Moreover, Pennington et al. (2002) [53] showed that maternal

237 preconceptional alcohol intake may be associated with hypertriglyceridemia in adult offspring

238 who have never been exposed to alcohol. Paternally inherited alterations in epigenetic

239 programming were also shown to be related to metabolic defects observed in foetal alcohol

240 spectrum disorders. In rodents, chronic paternal alcohol use affected insulin signaling and lipid

241 homeostasis in the offspring through paternally inherited alterations in liver x receptor activity

242 [28]. Similar findings were reported by Chang et al. (2019b) [29], assessing long-term impacts of

243 chronic preconception paternal alcohol use in mice. Evidence of metabolic programming in

244 offspring included suppressed cytokine profiles within the liver and pancreas, consistent with

245 findings from this study.

246 In our study, most of the tested hepatic biomarkers in the alcohol-naïve offspring of WHP

247 rats had changed similarly to the heavy alcohol abusers. Surprisingly, the serum activity of the 248 most specific alcohol-related biomarker, GGT, was decreased as compared to the WLP 249 counterparts and the Wistar controls. These contrary results are difficult to explain. GGT, unlike 250 AST and ALT, is located on the external surface of cellular membranes. Chronic alcohol use is 
251 considered to induce membrane GGT solubilization and increase the release of the enzyme, thus,

252 the mechanism of release of given liver enzymes is related to the degree of cell damage. One can

253 also hypothesise that different epigenetic mechanisms regulate the activity of these enzymes [54]

254 and one of the possible mechanisms could be linked to the changes in cellular membrane

255 properties. Nevertheless, the mechanism responsible for this is yet to be discovered.

256 The results of this study should be interpreted in light of certain limitations. WHP and

257 WLP line breeding involves the process of selecting rats in each generation through exposure to

258 alcohol. Therefore, the design of the study could not include a control group of the offspring of

259 WHP unexposed rats and, similarly, for this reason distinguishing between the paternal and

260 maternal effects was also unfeasible as both males and females were required to be exposed to

261 alcohol. Also, due to ethical concerns, we were only able to include a limited number of rats per

262 group, thus, only male offspring were examined. However, there are sex-specific effects on

263 offspring growth and long-term metabolic programming [29], thus, comparable studies on

264 female offspring are warranted.

265 It should be emphasized that although the offspring were not directly exposed to alcohol,

266 the last estrogenic cycle for females, as well as spermatogenesis in males, were exposed to

267 alcohol. Thus, it cannot be excluded that periconceptional exposure could have an impact on

268 gametes and affect, for example, oocyte maturation [55-57]. Similarly, this may influence the

269 perinatal behaviours of parents and food intake in such a way that it may have indirect effects on

270 metabolism. It has been shown, for example, that exposure to alcohol three weeks before

271 conception affects the birth weight and body growth of offspring [23]. In our study, however, we

272 failed to observe any differences in weight gain between the examined rat lines. 


\section{Conclusions}

274 In conclusion, the present study revealed that the alcohol-naïve offspring of high alcohol

275 preference rats differed significantly from their low alcohol preference counterparts and wild

276 Wistar rats in terms of common markers reflecting cardiovascular, liver, and pancreatic

277 disorders. To our knowledge, this is the first study showing the differences in selected

278 biochemical markers of metabolic disorders in high- and low-preferring rats. One can

279 hypothesise that these changes are associated with epigenetic changes at the level of the gametes

280 and/or with the genetically inherited phenotype. Either way, this study suggests that parental

281 alcohol abuse can have a detrimental influence on offspring vulnerability to metabolic disorders.

282 Given the detrimental influence of excessive alcohol consumption and metabolic 283 disorders on individuals and societies, the present findings should be verified in further 284 preclinical and clinical studies.

285

286 Acknowledgments

287 None.

288

289

References

1. Corrao G, Bagnardi V, Zambon A, La Vecchia C. A meta-analysis of alcohol consumption and the risk of 15 diseases. Prev Med. 2004;38:613-619.

2. Ronksley PE, Brien SE, Turner BJ, Mukamal KJ, Ghali WA. Association of alcohol consumption with selected cardiovascular disease outcomes: a systematic review and meta-analysis. Br Med J. 2011;342:d671. 
295 3. Yang AL, Vadhavkar S, Singh G, Omary MB. Epidemiology of alcohol-related liver and 296 pancreatic disease in the United States. Arch Intern Med. 2008;168:649-656.

297 4. Athyros VG, Liberopoulos EN, Mikhailidis DP, Papageorgiou AA, Ganotakis ES, 298 Tziomalos K, Kakafika AI, Karagiannis A, Lambropoulos S, Elisaf M. Association of 299 drinking pattern and alcohol beverage type with the prevalence of metabolic syndrome, 300 diabetes, coronary heart disease, stroke, and peripheral arterial disease in a 301 Mediterranean cohort. Angiology. 2007;58:689-697.

302 5. Alkerwi A, Boutsen M, Vaillant M, Barre J, Lair ML, Albert A, Guillaume M, Dramaix M.

303 Alcohol consumption and the prevalence of metabolic syndrome: a meta-analysis of 304 observational studies. Atherosclerosis. 2009;204:624-625.

305 6. Kahl KG, Hillemacher T. The metabolic syndrome in patients with alcohol dependency: 306 Current research and clinical implications. Prog Neuro-Psychopharmacol Biol Psychiatry. 2016;70:49-56.

308

7. Kendler KS, Heath AC, Neale MC, Kessler RC, Eaves LJ. A population-based twin study of alcoholism in women. $J$ Am Med Assoc. 1992;268:1877-1882.

8. Kendler KS, Davis CG, Kessler RC. The familial aggregation of common psychiatric and substance use disorders in the National Comorbidity Survey: a family history study. $\mathrm{Br} J$ Psychiatry. 1997;170:541-548.

9. Heath AC, Bucholz KK, Madden PA, Dinwiddie SH, Slutske WS, Bierut LJ, Statham DJ, Dunne MP, Whitfield JB, Martin NG. Genetic and environmental contributions to 315 alcohol dependence risk in a national twin sample: consistency of findings in women and men. Psychol Med. 1997;27:1381-1396. 
317 10. Anjos S, Polychronakos C. Mechanisms of genetic susceptibility to type I diabetes: beyond

$318 \quad$ HLA. Mol Genet Metab. 2004;81:187-195.

319 11. Joy T, Lahiry P, Pollex RL, Hegele RA. Genetics of metabolic syndrome. Curr Diabetes $320 \quad$ Rep. 2008;8:141-148.

321 12. Blakemore AI, Buxton JL. Obesity, genetic risk, and environment. $\mathrm{Br}$ Med $\mathrm{J}$. $322 \quad 2004 ; 348: \mathrm{g} 1900$.

323 13. Jones KL. The effects of alcohol on fetal development. Birth Defects Res, Part C. 2011;93:311.

14. Lange S, Probst C, Gmel G, Rehm J, Burd L, Popova S. Global Prevalence of Fetal Alcohol Spectrum Disorder Among Children and Youth: A Systematic Review and Metaanalysis.JAMA Pediatr. 2017;17:948-956.

15. Roozen S, Peters GJ, Kok G, Townend D, Nijhuis J, Curfs L.Worldwide prevalence of fetal alcohol spectrum disorders: A systematic literature review including metaanalysis.Alcohol Clin Exp Res. 2016;40:18-32.

16. Carter RC, Jacobson JL, Molteno CD, Dodge NC, Meintjes EM, Jacobson SW. Fetal alcohol growth restriction and cognitive impairment. Pediatrics. 2016;138:1-9.

17. Carter RC, Jacobson JL, Sokol RJ, Avison MJ, Jacobson SW. Fetal alcohol-related growth restriction from birth through young adulthood and moderating effects of maternal prepregnancy weight. Alcohol Clin Exp Res. 2013;37:452-62.

18. Moore EM, Riley EP. What happens when children with fetal alcohol spectrum disorders become adults? Curr Dev Disord Rep. 2015;2:219-27.

19. Gardejber EM, Cuffe JSM, Ward LC, Steane S, Anderson ST, Dorey ES, Kalish-Smith JI, 
Effects of periconceptional maternal alcohol intake and a postnatal high-fat diet on obesity and liver disease in male and female rat offspring. Am J Psysiol: Endocrinol Metab. 2018;315:E694-E704.

343

20. Dorey ES, Cullen CL, Lucia D, Mah KM, Manchadi MR, Muhlhausler BS, Moritz KM. The impact of periconceptional alcohol exposure on fat preference and gene expression in the mesolimbic reward pathway in adult rat offspring. $J$ Dev Originis Health Dis. 2018;9:223-231.

21. Knezovich JG, Ramsay M. The effect of preconception paternal alcohol exposure on epigenetic remodeling of the h19 and rasgrfl imprinting control regions in mouse offspring. Front Genet. 2012;3:10.

22. Finegersh A, Rompala GR, Martin DIK, Homanics GE. Drinking beyond a lifetime: New and emerging insights into paternal alcohol exposure on subsequent generations. Alcohol. 2015;49:461-470.

23. Jabbar S, Chastain LG, Gangisetty O, Cabrera MA, Sochacki K, Sarkar DK. Preconception Alcohol Increases Offspring Vulnerability to Stress. Neuropsychopharmacology. 2016;41:2782-2793.

24. Rompala GR, Homanics GE. Intergenerational Effects of Alcohol: A Review of Paternal Preconception Ethanol Exposure Studies and Epigenetic Mechanisms in the Male Germline. Alcohol: Clin Exp Res. 2019;43:1032-1045.

25. Kim P, Choi CS, Park JH, Joo SH, Kim HM, Ko HM, Kim KC, Jeon SJ, Park SH, Han SH, Ryu JH, Cheong JH, Han JY, Ko KN, Shin CY. Chronic exposure to ethanol of male mice before mating produces attention deficit hyperactivity disorder-like 
362 phenotype along with epigenetic dysregulation of dopamine transporter expression in 363 mouse offspring. J Neurosci Res. 2014;92:658-670.

364 26. Asimes A, Torasco A, Pinceti E, Kim CK, Zeleznik-Le NJ, Pak TR. Adolescent binge365 pattern alcohol exposure alters genome-wide DNA methylation patterns in the 366 hypothalamus of alcohol-naïve male offspring. Alcohol. 2017;60:179-189.

367 27. Przybycien-Szymanska MM, Rao YS, Prins SA, Pak TR. Parental Binge Alcohol Abuse 368 Alters F1 Generation Hypothalamic Gene Expression in the Absence of Direct Fetal Alcohol Exposure. PloS One. 2014;9:e89320.

28. Chang RC, Thomas KN, Bedi YS, Golding MC. Programmed increases in LXR $\alpha$ induced by paternal alcohol use enhance offspring metabolic adaptation to high-fat diet induced obesity. Molecular metabolism, 2019a. 30:161-172.

29. Chang RC, Wang H, Bedi Y, Golding MC. Preconception paternal alcohol exposure exerts sex-specific effects on offspring growth and long-term metabolic programming. Epigenetics Chromatin 2019b, 12(1):9.

30. Rok-Bujko P, Dyr W, Kostowski W. Operant self-administration of ethanol in Warsaw HighPreferring (WHP) and Warsaw Low-Preferring (WLP) lines of rats. Pharmacological Reports; 2006,58:931-935.

31. Dyr W, Kostowski W. Warsaw high-preferring (WHP) and Warsaw low-preferring (WLP) lines of rats selectively bred for high and low voluntary ethanol intake: preliminary phenotypic characterization. Alcohol. 2008;42:161-170.

32. Zakrzewska A, Kuczyńska J, Dermanowski M, Wyszogrodzka E, Dominiak M, of administration: Study on rats. Basic Clin Pharmacol Toxicol. 2020;126:51-58. 
385 33. Mikołajczak P, Okulicz-Kozaryn I, Kamińska E, Wiktorowicz K, Leśniewska K, Dyr W,

386 Kostowski W. Effect of subchronic ethanol treatment on plasma and cerebrospinal fluid

387 leptin levels in rats selectively bred for high and low alcohol preference. Pol J

$388 \quad$ Pharmacol. 2002;54:127-132.

389 34. Hynes GR, Jones PJ. Leptin and its role in lipid metabolism. Curr Opin Lipidol. $390 \quad 2001 ; 12(3): 321-327$.

391 35. Otaka M, Konishi N, Odashima M, Jin M, Wada I, Matsuhashi T, Ohba R, Watanabe S. 392 Effect of alcohol consumption on leptin level in serum, adipose tissue, and gastric mucosa. Dig Dis Sci,52 (2007)3066-3069.

394 36. Tan X, Sun X, Li Q, Zhao Y, Zhong W, Sun X, Jia W, McClain CJ, Zhou Z. Leptin 395 Deficiency Contributes to the Pathogenesis of Alcoholic Fatty Liver Disease in Mice. The American Journal of Pathology,181, 2012:1279-1286.

37. Lindenstrøm E, Boysen G, Nyboe J. Influence of total cholesterol, high density lipoprotein 398 cholesterol, and triglycerides on risk of cerebrovascular disease: the Copenhagen City Heart Study. Br Med J. 1994;309:11-15.

38. Langsted A, Freiberg JJ, Tybjaerg-Hansen A, Schnohr P, Jensen GB, Nordestgaard BG. Nonfasting cholesterol and triglycerides and association with risk of myocardial 402 infarction and total mortality: the Copenhagen City Heart Study with 31 years of follow-up. 403 J Intern $\quad$ Med. 2010;270:65-75.

404 39. Triglyceride Coronary Disease Genetics Consortium and Emerging Risk Factors 405 Collaboration, Sarwar N, Sandhu MS, Ricketts SL, Butterworth AS, Di Angelantonio E, Boekholdt SM, Ouwehand W, Watkins H, Samani NJ, Saleheen D, Lawlor D, Reilly MP, 

disease: collaborative analysis of 101 studies. Lancet. 2010;375:1634-1639.

40. Lawrence de Koning AB, Werstuck GH, Zhou J, Austin RC. Hyperhomocysteinemia and its role in the development of atherosclerosis. Clin Bioch. 2003;36:431-441.

411 41. Guthikonda S, Haynes WG. Homocysteine: role and implications in atherosclerosis. Curr Artheroscler Rep. 2006;8:100-106.

413 42. Van der Gaag MS, Ubbink JB, Sillanaukee P, Nikkari S, Hendriks HF. Effect of 414 consumption of red wine, spirits, and beer on serum homocysteine. Lancet. 2000; 355:1522.

415 43. Gibson A, Woodside JV, Young IS, Sharpe PC, Mercer C, Patterson CC, McKinley MC, study. QJM. 2008;101:881-887.

44. Bleich S, Degner D, Wiltfang J, Maler JM, Niedmann P, Cohrs S, Mangholz A, Porzig J, Sprung R, Rüther E, Kornhuber J. Elevated homocysteine levels in alcohol withdrawal. Alcohol. 2000;35:351-354.

45. Bleich S, Carl M, Bayerlein K, Reulbach U, Biermann T, Hillemacher T, Bönsch D, Kornhuber J. Evidence of increased homocysteine levels in alcoholism: the Franconian alcoholism research studies (FARS). Alcohol: Clin Exp Res. 2005;29:334-336.

46. Grucza RA, Krueger RF, Racette SB, Norberg KE, Hipp PR, Bierut LJ. The emerging link 426 between alcoholism risk and obesity in the United States. Arch Gen Psychiatry. 2010;67:1301-1308. 
428 47. Carr LG, Kimpel MW, Liang T, McClintick JN, McCall K, Morse M, Edenberg HJ.

429 Identification of candidate genes for alcohol preference by expression profiling of $430 \quad$ congenic rat strains. Alcohol: Clin Exp Res. 2007;31:1089-1098.

431 48. Kimpel MW, Strother WN, McClintick JN, Carr LG, Liang T, Edenberg HJ, McBride WJ. 432 Functional gene expression differences between inbred alcohol-preferring and non433 preferring rats in five brain regions. Alcohol. 2007;41:95-132

49. Stankiewicz AM, Goscik J, Dyr W, Juszczak GR, Ryglewicz D, Swiergiel AH, Wieczorek

M, Stefanski R. Novel candidate genes for alcoholism - transcriptomic analysis of prefrontal medial cortex, hippocampus and nucleus accumbens of Warsaw alcohol-preferring and non-preferring rats. Pharmacol Biochem Behav. 2015;139:27-38.

50. Shukla SD, Velazquez J, French SW, Lu SC, Ticku MK, Zakhari S. Emerging Role of Epigenetics in the Actions of Alcohol. Alcohol: Clin Exp Res. 2008;32:1525-1534.

51. Mahnke AH, Miranda RC, Homanics GE. Epigenetic Mediators and Consequences of Excessive Alcohol Consumption. Alcohol. 2017;60:1-6.

52. Chastain LG, Sarkar DK. Alcohol effects on the epigenome in the germline: role in the inheritance of alcohol-related pathology. Alcohol. 2017;60:53-66.

53. Pennington JS, Shuvaeva T, Pennington SN. Maternal dietary ethanol consumption is associated with hypertriglyceridemia in adult rat offspring. Alcohol: Clin Exp Res.

54. Teschke R. Alcoholic Liver Disease: Alcohol Metabolism, Cascade of Molecular Mechanisms, Cellular Targets, and Clinical Aspects. Biomedicines. 2018;6:106. Eckert JJ. The embryo and its future. Biol Reprod. 2004;71(4):1046-1054. 
451 56. Bielawski DM, Zaher FM, Svinarich DM, Abel EL. Paternal alcohol exposure affects sperm cytosine methyltransferase messenger RNA levels. Alcohol Clin Exp Res. 2002;26:347453 351.

454 57. Kalisch-Smith J, Steane SE, Simmons DG, Pantaleon M, Anderson ST, Akison LK, Wlodek 455 ME, Moritz KM. Periconceptional alcohol exposure causes female-specific perturbations t o trophoblast differentiation and placental formation in the rat. Development 2019;146:dev172205. 


\section{Table $\mathbf{1}$ (on next page)}

Biochemical characteristics of the alcohol-naïve offspring of WHP, WLP and alcoholnaïve wild Wistar rats.

*offspring of WHP rats vs offspring of WLP rats; ${ }^{*}$ offspring of WHP rats vs Wistar rats;

${ }^{\dagger}$ offspring of WLP rats vs Wistar rats; values represent mean (SD); p-value from NewmanKeuls test or Dunn's test (for ALT and triglycerides) 
1 Table 1. Biochemical characteristics of the alcohol-naïve offspring of WHP, WLP and alcohol2 naïve wild Wistar rats.

\begin{tabular}{|c|c|c|c|c|}
\hline Parameters & WHP $(n=8)$ & WLP $(n=8)$ & Wistar $(n=8)$ & P-value \\
\hline $\begin{array}{l}\text { Homocysteine } \\
(\mu \mathrm{mol} / \mathrm{L})\end{array}$ & $7.48(1.37)^{* \#}$ & $4.77(1.55)$ & $6.57(0.68)$ & $* \mathrm{p}<0.001 ;{ }^{*} \mathrm{p}=0.009$ \\
\hline Glucose (mg/dL) & $143.5(13.55)$ & $149.83(12.11)$ & $143.16(8.03)$ & all $\mathrm{p}>0.05$ \\
\hline $\begin{array}{l}\text { Total cholesterol } \\
(\mathrm{mg} / \mathrm{dL})\end{array}$ & $79.37(16.30)^{* \#}$ & $34.87(3.87)^{\dagger}$ & $46.00(2.72)$ & ${ }^{*} \mathrm{p}<0.001 ;{ }^{*} \mathrm{p}<0.001 ;{ }^{\dagger} \mathrm{p}=0.033$ \\
\hline $\begin{array}{l}\text { Triglycerides } \\
(\mathrm{mg} / \mathrm{dL})\end{array}$ & $74.28(22.94)^{* \#}$ & $25.06(4.63)$ & $37.91(2.47)$ & ${ }^{*} \mathrm{p}<0.001 ;{ }^{\#} \mathrm{p}<0.001$ \\
\hline $\operatorname{ALT}(\mathrm{U} / \mathrm{L})$ & $97.75(13.18)^{* \#}$ & $74.25(7.95)^{\dagger}$ & $63.81(5.18)$ & ${ }^{*} \mathrm{p}=0.04 ;{ }^{*} \mathrm{p}<0.001 ;{ }^{\dagger} \mathrm{p}=0.02$ \\
\hline AST (U/L) & $200.5(22.78)^{\#}$ & $177.66(29.34)$ & $152.53(25.87)$ & ${ }^{*} \mathrm{p}=0.004$ \\
\hline GGT (U/L) & $4.95(0.74)^{* \#}$ & $9.85(0.54)$ & $9.67(0.72)$ & $* \mathrm{p}<0.001 ;{ }^{*} \mathrm{p}<0.001$ \\
\hline Amylase (U/L) & $2174.37(359.72)^{\#}$ & $2073.25(189.26)^{\dagger}$ & $1559.37(131.57)$ & ${ }^{\#} \mathrm{p}<0.001 ;{ }^{\dagger} \mathrm{p}<0.001$ \\
\hline
\end{tabular}

3

4 *offspring of WHP rats vs offspring of WLP rats; * offspring of WHP rats vs Wistar rats;

5 toffspring of WLP rats vs Wistar rats;

6 values represent mean (SD); p-value from Newman-Keuls test or Dunn's test (for ALT and

7 triglycerides) 